\title{
BMJ Open Quality Prevention of acute kidney injury through accurate fluid balance monitoring
}

\section{Andrew Davies, ${ }^{1}$ Seema Srivastava, ${ }^{2}$ William Seligman, ${ }^{1}$ Lorraine Motuel, ${ }^{2}$ Vardeep Deogan, ${ }^{2}$ Shaza Ahmed, ${ }^{3}$ Nicholas Howells ${ }^{1}$}

To cite: Davies A, Srivastava S, Seligman W, et al. Prevention of acute kidney injury through accurate fluid balance monitoring.BMJ Open Quality 2017;6:e000006. doi:10.1136/ bmjoq-2017-000006

- Additional material is published online only. To view please visit the journal online (http://dx.doi.org/10.1136/ bmjoq-2017-000006).

Received 9 January 2017 Revised 26 September 2017 Accepted 27 September 2017

\section{ABSTRACT}

Acute kidney injury (AKI) is associated with increased patient morbidity, mortality and an extended hospital stay. The financial burden to the National Health Service is high and it can affect up to one in five inpatients. Optimal fluid balance management is essential for the prevention of AKI and this can be particularly challenging in the patient with trauma. Our aim was to reduce the rate of AKI in patients with traumatic injuries in the regional trauma centre. We developed new fluid balance charts and documented how well these were completed. The number of AKI alerts per month was calculated on our pathology system. Scenario training was delivered at handover meetings and an e-learning tool was designed at three levels: healthcare assistants; nurses; and medical staff, dietetics and pharmacists. Educational posters were placed in clinical areas and patient information leaflets produced. Junior doctors were regularly informed of AKI rates on the ward. The number of AKI alerts on our trauma ward declined from 50 in January 2016 to 19 in November 2016. The mean monthly rate of AKI fell $33 \%$ following the invention $(\mathrm{P}<0.001)$. Completion of fluid balance charts improved; 6 hourly urine output documentation increased from $36 \%$ to $68 \%$ and running 1 hourly output increased from $80 \%$ to $96 \%$. Calculation of total daily fluid balance rose from $12 \%$ to $72 \%$, before decreasing to $32 \%$. This highlighted the need for continued encouragement. Improved fluid balance monitoring led to a reduction in the prevalence of AKI in patients admitted to this trauma centre.

\section{PROBLEM}

Acute kidney injury (AKI) is a common condition among hospital inpatients. It is associated with increased morbidity and mortality and an extended length of stay. ${ }^{12}$ AKI costs the National Health Service over a billion pounds annually. $^{2}$ This regional trauma centre and tertiary referral arthroplasty centre has a dedicated orthopaedic trauma ward that receives the new patients and several mixed trauma rehabilitation and elective wards.

Patients admitted following acute traumatic injuries are at particular risk of AKI. ${ }^{3}$ In common with Trusts nationally, our electronic blood test result reporting system includes an 'AKI alert' with the biochemistry results when the patient's creatinine rises over a certain threshold. AKI is defined at our Trust as an increase of serum creatinine of $\geq 26 \mu \mathrm{mol} / \mathrm{L}$ within 48 hours or an increase in serum creatinine $\geq 1.5$ times baseline. This is consistent with international guidelines. ${ }^{4}$ The pathology system grades the severity of AKI as 1, 2 or 3:

Grade 1: Increase of serum creatinine of $\geq 26 \mu \mathrm{mol} / \mathrm{L}$ within 48 hours or an increase in serum creatinine $\geq 1.5$ to 1.9 times baseline.

Grade 2: Increase in serum creatinine $\geq 2$ to 2.9 times baseline.

Grade 3: Increase in serum creatinine $\geq 3.0$ times baseline or $\geq 44 \mu \mathrm{mol} / \mathrm{L}$ rise when serum creatinine is $\geq 354 \mu \mathrm{mol} / \mathrm{L}$.

If an alert is triggered, this is stored on the system database and a Trust management protocol is provided alongside the result based on Trust guidelines. The recommendations depend on the severity of the alert. The database can be accessed at any time to determine the number of AKI alerts in a given period.

Fluid balance monitoring is fundamental to both the diagnosis and successful management of this condition. However, documentation of fluid balance on our orthopaedic wards was limited. An initial audit on the primary trauma ward revealed only $12 \%$ of patients with fluid charts had a running 24 hourly total fluid balance documented. Although patient weights are important for calculating relative urine output, they were stated on only $8 \%$ of the charts and a 6 hourly output was noted on $36 \%$.

The mean number of monthly AKI alerts from October 2015 to January 2016 was 47. Our aim was to achieve a $50 \%$ reduction in the total number of AKI alerts each month between January 2016 and November 2016. We targeted the accuracy of fluid balance documentation and encouraged staff to act promptly on any decrease in output or abnormality in fluid balance. 


\section{BACKGROUND}

There is large variation in the fluid requirements of patients following acute trauma and during the perioperative period. ${ }^{5}$ Optimising fluid management can lead to improved outcomes, and AKI may occur if this is not achieved. ${ }^{67}$ Caution is necessary as patients are often volume deplete following acute trauma; however, overzealous intravenous fluid resuscitation may lead to fluid overload and worsen an AKI despite poor urine output in this patient group. ${ }^{8}$ As a result, accurate fluid balance monitoring from the time of admission is necessary to guide resuscitation following trauma and during the perioperative period.

\section{BASELINE MEASUREMENT}

Data collection focused on the two central components: the thoroughness of documentation on our fluid balance charts and the total number of AKI alerts raised on our electronic reporting system.

Six key components of the fluid balance charts were selected:

1. Patient details.

2. Patient weight.

3. Running hourly input.

4. Six hourly input subtotal.

5. Six hourly output subtotal.

6. 24-hour total fluid balance.

A proforma was created and data collected from five fluid balance charts each day on our acute trauma ward for 5 days. This provided a total of 25 charts for each of the three cycles. Nursing staff on the ward responsible for completing the patients' charts were not aware of which charts would be audited. The same fluid balance chart was not checked on consecutive days. The total number of AKI alerts was collected each month and the Exact Poisson Test performed on the difference between the rate of AKI before and after the interventions. The total number of alerts was chosen as an outcome measure over the number of new cases of AKI because this incorporated those patients where AKI recurred, a problem which we aimed to prevent. Trauma ward logs were reviewed to determine the number of new ward admissions each month.

Our initial results demonstrated variable chart completion. One hundred per cent included patient details; however, only $8 \%$ had a documented patient weight. Running 1-hour input was completed on $80 \%$ and 6-hour input on $83 \%$. Data on urine output include 6-hour totals in $36 \%$ and 24 -hours balance in $12 \%$. The mean monthly total number of AKI alerts for the 4 months prior to commencing our interventions was 47 (see online supplementary file 1 - New fluid balance chart').

\section{DESIGN}

Given the complexity of managing AKI, the challenges of accurately documenting fluid balance and the breadth of clinical staff involved, we focused our interventions on several groups in the multi disciplinary team. We redesigned the fluid balance monitoring charts so they were simple, clear, uncluttered and highlighted our six key components. The design of the new fluid balance charts was undertaken by a multidisciplinary group including anaesthetists, ortho-geriatricians, orthopaedic surgeons, nurses and healthcare assistants; the charts are used throughout the patient journey and may be started in the anaesthetic rooms. In order to embed the charts into clinical practice, we promoted them at nursing handovers and included discussion about the importance of fluid balance monitoring. We ensured all junior doctors responsible for reviewing these charts were informed of the new development. Subsequent interventions were introduced where we recognised limitations or restrictions to change.

\section{STRATEGY}

Several weeks after the introduction of the charts, we noted a series of common errors. These included comments such as 'OTT' or 'wet' in the output section. In certain cases, completion was excellent and we wished to highlight this. Our baseline data collection was carried out in January 2016. Posters were introduced following PDSA cycle one in February 2016 with examples of correct and incorrect charts. These were placed them in clinical areas, staff were directed to these as a reference. Challenges were discussed at nursing handover meetings.

The response to our initial interventions was assessed during cycle two; fluid balance charts were reaudited in March 2016. Despite improvement, we were concerned about a gradual reduction in engagement with the new charts. To reverse this trend and consolidate the education of ward staff, during the second plan do study act (PDSA) cycle we created e-learning modules at three levels: those aimed at (1) Health Care Assistants (HCAs); (2) staff nurses; (3) senior staff nurses and ward sisters. The e-learning modules were designed and written by a consultant renal physician with support from the Trust information technology team. The content of the modules aligned with national guidelines on fluid management and AKI. ${ }^{9} 10$ This included information about fluid prescriptions, potential complications and how to manage them. The modules were distributed to all staff and are available through the trust intranet. We did not formally assess learners' knowledge following this intervention; however, an MCQ test was included at the end of the e-learning modules to assess retention. The education has extended to patients and we have developed a Trust information leaflet, distributed to those patients with a new AKI, which includes details on how to manage fluid intake.

Fluid balance charts were reaudited in October 2016. To assess the ongoing prevalence of AKI in parallel with the PDSA cycles, the number of AKI alerts was monitored on a monthly basis. 
Junior doctors were central to driving change. We currently have 20 foundation doctors and 'SHO' level trainees and fellows in our department. We are able to monitor the number of AKI alerts on a daily basis, which provides the opportunity to update the team about AKI numbers on the ward and flag up any increase in alerts. We regularly disseminated any increase in alert numbers to the trauma teams and encouraged close monitoring of the fluid balance charts. This has been particularly relevant during handover periods as new juniors rotate into the department. During late August and September, there was a notable increase in alerts. This is likely to be multifactorial; however, due to the results reporting system, we were able to disseminate this information quickly.

\section{RESULTS}

\section{Acute kidney injury alerts}

The number of alerts each month on our primary trauma ward declined from 50 in January 2016 to 19 in our most recent data set from November 2016. Our system provides alert data from September 2015. The mean monthly alert for the 4 months prior to commencing our interventions was 47 (range 42-50). This declined by 33\% (95\% CI 20\% to $45 \%$, Exact Poisson Test $\mathrm{P}$ value $<0.001$ ) to a mean of 31 (range 17-65) for the 10 months since initiating the project and continues to decline.

The mean number of new ward admissions each month for the 4 months prior to the intervention was 90 (range 78-93) and 84 (range 72-98) postintervention.

\section{Fluid balance monitoring charts}

The percentage of charts with the running 1-hour total completed increased from $80 \%$ to $96 \%$. The 6 hourly output increased from $36 \%$ to $68 \%$. However, the improvement in patient weights and running totals were more modest. The percentage of charts with patient weight completed initially improved from $8 \%$ to $80 \%$ before falling back to $16 \%$. Running totals rose from $12 \%$ to $72 \%$ and subsequently fell to $32 \%$. One of the comments from staff was the difficulty in quickly adding up the total input and output and providing a 24-hours total (see online supplementary file 2-'Results').

\section{Lessons and limitations}

Prevention and the optimal management of AKI are the responsibility of the whole multidisciplinary team and several strategies are necessary to reduce the incidence of this condition. Over the last year, we have noted an increased vigilance towards fluid balance and urine output during daily ward reviews. Without accurate data, AKI prevention is more difficult. It is common practice on all hospital ward rounds to scrutinise the patient's observation charts, and doctors are alerted to patients whose 'early warning score' rises above a certain level. However, this early warning score does not currently include fluid balance or urine output.

Documentation remains a time-consuming process during busy ward shifts, and calculating 24 hourly total balances is an additional task. However, staff education and demonstration of improvement in AKI incidence may provide additional motivation.

A potential limitation is that we did not record the total number of renal function tests performed on each ward during the assessment period, which may have affected the number of AKI alerts. However, the number of new admissions each month was similar across the study period and there has been no change to our policy of perioperative renal function monitoring. Due to everpresent bed pressures, the 32-patient ward is very rarely at less than full capacity.

Going forward we aim to extend the project to optimise our management of AKI. Previous studies have documented different approaches to this. For example, Forde et al describe an ABCDE checklist: Address drugs, Boost blood pressure, Calculate fluid balance, Dip urine, Exclude obstruction. ${ }^{11}$ Bhagwanani et al propose the DONUT bundle: Dehydration, Obstruction, Nephrotoxins, Urine, Think sepsis. ${ }^{12}$ The nephrology department at our Trust has produced an assessment protocol, against which we will audit our performance.

The impact of this work is not limited to patients with trauma; our elective orthopaedic patients are now benefiting from our new fluid balance charts, all staff have access to the e-learning modules and the patient information leaflet is available across the Trust. The preoperative assessment clinics for elective patients provide an opportunity for staff to highlight those at particular risk of AKI. The charts are commenced preoperatively and we aim to develop a preoperative scoring system and perioperative management protocol for high-risk groups.

\section{CONCLUSION}

Increased vigilance towards patient fluid balance was associated with a reduction in the incidence of AKI in this trauma centre. Engagement from all members of the multidisciplinary ward team was necessary for sustained improvement. Our new monitoring charts have been started on all our orthopaedic wards and will be distributed across the Trust.

\section{Acknowledgements Thanks to Helen Jones, Eva Stacey and Hannah Mayall.}

Contributors AD: project design, data collection, data analysis, intervention design and implementation, write-up. SS: project design, data analysis, review of manuscript, promotion of project. WS: data collection, data analysis, review of manuscript. LM: data collection, intervention design and implementation, review of manuscript. VD: intervention design and implementation, review of manuscript, promotion of project. SA: data collection, data analysis, review of manuscript, intervention design and implementation. $\mathrm{NH}$ : project design, data analysis, review of manuscript, promotion of project, supervision of project.

\section{Competing interests None declared.}

Provenance and peer review Not commissioned; externally peer reviewed.

Open Access This is an Open Access article distributed in accordance with the Creative Commons Attribution Non Commercial (CC BY-NC 4.0) license, which permits others to distribute, remix, adapt, build upon this work non-commercially, and license their derivative works on different terms, provided the original work is properly cited and the use is non-commercial. See: http://creativecommons.org/ licenses/by-nc/4.0/ 
(c) Published by the BMJ Publishing Group Limited. For permission to use (where not already granted under a licence) please go to http://www.bmj.com/company/ products-services/rights-and-licensing/

\section{REFERENCES}

1 National Confidential Enquiry into Patient Outcome and Death (NCEPOD). Adding Insult to Injury. A review of the care of patients who died in hospital with a primary diagnosis of acute kidney injury (acute renal failure), 2009. https://www.ncepod.org.

2 Kerr M, Bedford M, Matthews B, et al. The economic impact of acute kidney injury in England. Nephrol Dial Transplant 2014;29:1362-8.

3 Bihorac A, Delano MJ, Schold JD, et al. Incidence, clinical predictors, genomics, and outcome of acute kidney injury among trauma patients. Ann Surg 2010;252:158-65.

4 Khwaja A. KDIGO clinical practice guidelines for acute kidney injury. Nephron Clin Pract 2012;120:c179-c184.

5 Pearse RM, Ackland GL. Perioperative fluid therapy. BMJ 2012;344:e2865.
6 Strunden MS, Heckel K, Goetz AE, et al. Perioperative fluid and volume management: physiological basis, tools and strategies. Ann Intensive Care 2011;1:2.

7 Godin M, Bouchard J, Mehta RL. Fluid balance in patients with acute kidney injury: emerging concepts. Nephron Clin Pract 2013:123:238-45.

8 Bouchard J, Soroko SB, Chertow GM, et al. Program to Improve Care in Acute Renal Disease (PICARD) Study Group. Fluid accumulation, survival and recovery of kidney function in critically ill patients with acute kidney injury. Kidney Int 2009;76:422-7.

9 National institute for Health and Care Excellence (2013) Intravenous fluid therapy in adults in hospital. NICE guideline (CG174), 2013.

10 National institute for Health and Care Excellence (2013) Acute kidney injury: prevention, detection and management. NICE guideline (CG169), 2013.

11 Forde C, McCaughan J, Leonard N. Acute Kidney Injury: It's as easy as ABCDE. BMJ Qual Improv Rep 2012;1:u200370.w326.

12 Bhagwanani $A$, Carpenter R, Yusuf $A$. Improving the management of Acute Kidney Injury in a District General Hospital: Introduction of the DONUT bundle. BMJ Qual Improv Rep 2014;2:u202650.w1235. 